\title{
Pulmonary alveolar microlithiasis complicated by corpulmonale
}

\author{
G G A Gayani ${ }^{1}$, S Muhunthan ${ }^{2}$, A Perera ${ }^{3}$, C K Bodinayake ${ }^{4}$, M R Mohideen ${ }^{5}$
}

Journal of the Ceylon College of Physicians, 2013, 44, 40-41

\begin{abstract}
Pulmonary alveolar microlithiasis is a rare, chronic lung disease with bilateral intra-alveolar calcium and phosphate deposition throughout the lung parenchyma. This is a case history of a 40year-old female with a family history of lung disease, presenting with progressive respiratory symptoms for 5 years. She had clinical and radiological features of pulmonary alveolar microlithiasis and responded poorly to medical treatment, subsequently admitted with severe respiratory insufficiency, pulmonary hypertension and right heart failure. She died after repeated attempts at resuscitation. Her autopsy revealed atherosclerotic pulmonary arteries with intra alveolar deposits of concentrically laminated calcific bodies which confirmed the diagnosis of pulmonary alveolar microlithiasis. The disease evolved in a progressive protracted course with respiratory insufficiency which led to corpulmonale.
\end{abstract}

Key words: pulmonary alveolar microlithiasis, laminated calcipherities, corpulmonale.

\section{Introduction}

Pulmonary alveolar microlithiasis is a rare pulmonary disease of unknown pathogenesis characterized by widespread laminated calcipherites in alveolar spaces in the absence of any known disorder of calcium metabolism. It usually occurs in sporadic form, but also tend to occur in families with autosomal recessive pattern of inheritance. Very few cases have been reported in Sri Lanka ${ }^{1,2}$.

\section{Case report}

A 40-year-old female presented with worsening of dyspnoea and bilateral massive lower limb swelling

\footnotetext{
${ }^{1}$ Registrar in Medicine, University Medical Unit,

${ }^{2}$ Consultant Chest Physician,

${ }^{3}$ Consultant Histopathologist, Teaching Hospital Karapitiya, Galle, Sri Lanka.

${ }^{4}$ Consultant Physician, Senior Lecturer in Medicine,

${ }^{5}$ Professor in Medicine, Department of Medicine, Faculty of Medicine, University of Ruhuna, Galle, Sri Lanka.
}

for two months. She has had progressively worsening exertional dyspnoea of five years duration. She had chronic cough with whitish sputum, intermittent hemoptysis and loss of appetite. She also had upper backache and developed bilateral ankle swelling in the six months prior to presentation.

Her younger brother had died of a respiratory disease at the age of 29 years. There is no consanguinity.

On physical examination, she had a BMI of $12.6 \mathrm{~kg} / \mathrm{m}^{2}$, scoliosis and grade 4 clubbing. She was tachypnoeic, centrally cyanosed with a $\mathrm{SaO}_{2}$ of $84 \%$ on air and had elevated jugular venous pressure. There were bilateral diffuse fine inspiratory crepitations and a loud second heart sound on auscultation with evidence of pulmonary hypertension.

Her FBC, ESR, serum calcium were normal in range. Arterial blood gas analysis showed $\mathrm{pH}-7.48$, $\mathrm{PaO} 2$ 60mmHg, $\mathrm{PaCO} 2$ 38mmHg. Chest radiograph revealed diffuse high density micronodules and reticular lines obliterating bronchovascular bundles, mediastinal margins and diaphragm (Figure 1).

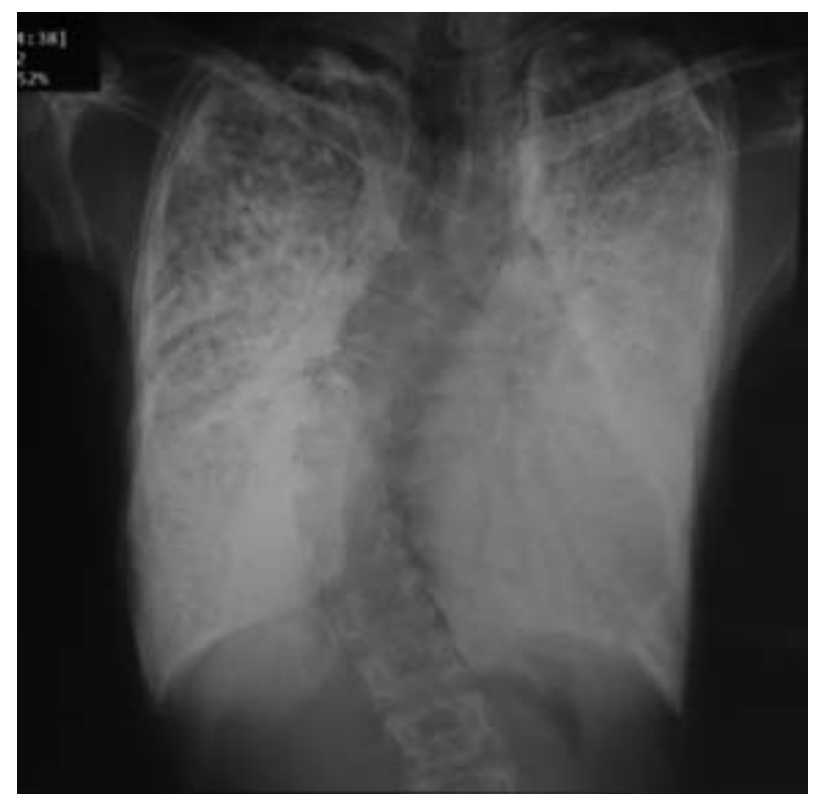

Figure 1. Chest radiograph showing high density opacities and reticular lines. 
Lung function tests revealed a severe restrictive pattern abnormality. Echocardiogram showed pulmonary hypertension with dilated mean pulmonary artery, right atrium and right ventricle. There was no evidence of intra-atrial or intra-ventricular shunts. High resolution CT of the chest revealed fine nodular calcific densities scattered throughout both lung fields along the peribronchiolar, subpleural and mediastinal pleural aspects suggestive of pulmonary alveolar microlithiasis. Traction bronchiectasis was seen in apical region of both lungs (Figure 2). Bronchoscopy showed calcium deposits in tracheal rings and bronchial cartilages.

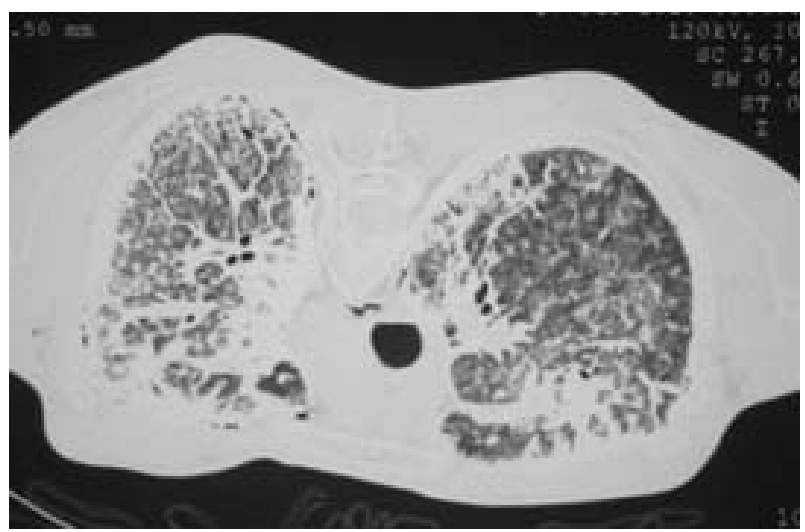

Figure 2. HRCT showing fine nodular calcific densities.

She was managed with high flow oxygen and was on a long-acting beta ${ }_{2}$ agonist and steroid inhalers and oral and intravenous bisphosphonates over three years. But gradually she developed progressive respiratory failure with pulmonary hypertension. Ultimately she had cardiorespiratory arrest and passed away despite resuscitation. Autopsy revealed heavy lungs $(3.5 \mathrm{~kg})$ with a gritty surface resembling multitude of scattered small stones. Liver showed nutmeg appearance due to congestive cardiac failure. Atherosclerotic plaques were seen in pulmonary artery. Microscopy revealed numerous wavy concentrically laminated microliths filling the alveoli and delicate fibrosis of alveolar wall. There was no calculous disease in other organs. The clinical, radiological and histological features confirmed the diagnosis of pulmonary alveolar microlithiasis (PAM).

\section{Discussion}

Pulmonary alveolar microlithiasis is diagnosed with clinical, radiological and histological features. PAM has been reported from 51 countries and $40.6 \%$ of these are reported from Asia $^{3}$ while $42.7 \%$ is reported from Europe. Mean age of presentation is 35 years ${ }^{4}$. Familial and sporadic form of PAM has been described and there is female predominance in the autosomal recessive familial form ${ }^{5}$. Most patients are asymptomatic and are found incidentally from chest radiographs $^{6}$. Cough is the commonest nonspecific symptom with very little expectoration at the beginning and haemoptysis is a rare presentation. Occasionally microliths may be coughed out. In later stages they may develop progressive pulmonary insufficiency leading to corpulmonale ${ }^{7}$. Diagnosis of PAM is made by bronchoalveolar lavage or open lung biopsy. Typical minute sandstorm opacities in HRCT are suggestive of $\mathrm{PAM}^{8}$.

Only curative treatment is bilateral sequential lung transplantation. Pharmacological measures are not promising and bisphosphonates such as disodium etidronate which is known to inhibit microcrystal growth of hydroxyapatite has been used in some cases without any evidence for proven benefit.

\section{References}

1. Balasuriya BMAC, Kularathna SAM, Ratnatunga N. A case report of pulmonary alveolar microlithiasis. Ceylon Medical Journal 2004; 49 (3): 91-2.

2. Kannagara RD, Ragunathan MK, Anoma Perera. Aregnant mother with pulmonary alveolar microlithiasis. Galle Medical Journal 2006; 11 (1): 53-4.

3. Mariotta S, Rocci A, Papale M, Declementi F, Spasato B, et al. Pulmonary alveolar microlithiasis: report on 576 cases published in the literature. Sarcoidosis, Vasculitis and Diffuse Lung Disease 2004; 21 (3): 173-81.

4. Senyigh A, Yaramis A, Gurkan F, Kirbas G, Buyukbarayan $\mathrm{H}$, et al. Pulmonary alveolar microlithiasis: a rare familial inheritance with report of 6 cases in family. Respiration 2001; 68 (2): 204-9.

5. Castellana G, Lamorgese V. Pulmonary alveolar microlithiasis. World cases and review of the literature. Respiration 2003; 70: 549-55.

6. Giuseppe Barbolini, Giulio Rossi, Alberto Bisetti. Pulmonary alveolar microlithiasis. New England Journal of Medicine 2002; 347: 69-70.

7. John G Ledingham, David A Warrell. Pulmonary alveolar microlithiasis. Concise Oxford Textbook of Medicine, 4th Ed, chapter 4.32, New York: Oxford University Press, 2000: 454.

8. Sumikawa H, Johkoh T, Tomiyama N, Hamada S, Koyoma M, Tsubamoto M, et al. Pulmonary alveolar microlithiasis: CT and pathologic findings in 10 patients. Arch Chest Disease 2005; 63: 59-64. 